\title{
Isotropic Stars in Higher-Order Torsion Scalar Theories
}

\author{
Gamal G. L. Nashed ${ }^{1,2}$ \\ ${ }^{1}$ Centre for Theoretical Physics, The British University in Egypt, P.O. Box 43, El-Sherouk City 11837, Egypt
}

${ }^{2}$ Mathematics Department, Faculty of Science, Ain Shams University, Cairo 11566, Egypt

Correspondence should be addressed to Gamal G. L. Nashed; nashed@bue.edu.eg

Received 20 September 2015; Revised 1 January 2016; Accepted 5 January 2016

Academic Editor: Chao-Qiang Geng

Copyright (C) 2016 Gamal G. L. Nashed. This is an open access article distributed under the Creative Commons Attribution License, which permits unrestricted use, distribution, and reproduction in any medium, provided the original work is properly cited. The publication of this article was funded by $\mathrm{SCOAP}^{3}$.

Two different nondiagonal tetrad spaces reproducing spherically symmetric spacetime are applied to the field equations of higherorder torsion scalar theories. Assuming the existence of conformal Killing vector, two isotropic solutions are derived. We show that the first solution is not stable while the second one confirms a stable behavior. We also discuss the construction of the stellar model and show that one of our solutions is capable of such construction while the other is not. Finally, we discuss the generalized Tolman-Oppenheimer-Volkoff and show that one of our models has a tendency to equilibrium.

\section{Introduction}

It is well known that in $f(T)$ gravity not only inflation [1] in the early universe but also late time cosmic acceleration can be realized [2-27]. Recently, there are many models constructed to describe dark energy without the use of cosmological constant (for more details, see review [28] and references therein). The main merit of $f(T)$ gravity is that its gravitational field equation is second order as GR. There are arguments in terms of theoretical properties of $f(T)$ gravity, for example, local Lorentz invariance [29-31], nonminimal coupling of teleparallel gravity to a scalar field [32-34], and nonlinear causality [35]. Recently, number of $f(T)$ gravitational theories have been proposed [36-62]. The structures of neutron and quark stars in $f(T)$ theory of gravity have been investigated [63]. The anisotropic behavior, regularity conditions, stability, and surface redshift of the compact stars have been checked [64]. Under those theories, it is shown that $f(T)$ are not dynamically identical to teleparallel action plus a scalar field [61]. It has been shown that investigations of $f(T)$, using observational data, are compatible with observations (see, e.g., $[65,66]$ and references therein). A new type of $f(T)$ theory was proposed in order to explain the acceleration phase of the universe [60]. Also, it has been shown that the well-known problem of frame dependence and violation of local Lorentz invariance in the formulation of $f(T)$ gravity is a consequence of neglecting the role of spin connection [31].

$f(T)$ theory coupled with anisotropic fluid has been examined for static spacetimes with spherical symmetry and many classes of solutions have been derived [67]. It has been shown that some conditions on the coordinates, energy density, and pressures can produce new classes of anisotropic and isotropic solutions. Some of new black holes and wormholes solutions have been derived by selecting a set of nondiagonal tetrads [68]. It has been shown that relativistic stars can exist in the frame of $f(T)$ and static spherically symmetric perfect fluid solutions have been derived [69]. A special analytic vacuum spherically symmetric solution with constant torsion scalar, within the framework of $f(T)$, has been derived [70]. D-dimensional charged flat horizon solutions have been derived for a specific form of $f(T)$; that is, $f(T)=T+\alpha T^{2}[71]$. A complete investigation of the Noether symmetry approach in $f(T)$ gravity at FRW and spherical levels, respectively, has been investigated [72]. In the framework of $f(T)$ gravitational theories, there are many solutions, spherically symmetric [59], spherically symmetric charged [73], homogenous anisotropic [74], and stability of the Einstein static closed and open universe [75]. Some cosmological features of the $\Lambda$ CDM model in the framework of the $f(T)$ are investigated [76]. However, till now, no spherically symmetric 
isotropic solution, using nondiagonal tetrad fields, is derived in this theory. It is the aim of the present study to find an analytic, isotropic spherically symmetric solution in higherorder torsion scalar theories. The arrangement of this study is as follows: in Section 2, ingredients of $f(T)$ gravitational theory are provided. In Section 3, two different tetrad spaces having spherical symmetry are applied to the field equations of $f(T)$. Assuming the conformal Killing vector (CKV), we derived two nonvacuum spherically symmetric solutions in Section 3. The physics relevant to the derived solutions are analyzed in Section 4. The energy conditions are satisfied for the two solutions provided that the constants of integration are positive. In addition, the stability condition, the nature of the star, and Tolman-Oppenheimer-Volkoff (TOV) equation are shown to be satisfied for one solution. The results obtained in this study are discussed in final section.

\section{Ingredients of $f(T)$ Gravitational Theory}

Another description of Einstein's general relativity (GR) of gravitation is done through the employment of what is called teleparallel equivalent of general relativity (TEGR). The ingredient quantity of this theory is the vierbein (tetrad) fields (Greek letters $\alpha, \beta, \ldots$ indicate spacetime indices while Latin letters $a, b, \ldots$ running from 0 to 3 describe Lorentz indices. Time and space indices are denoted as $\mu=0, i$ and $a=$ (0), (i), where $i=1,2,3$.) $\left\{h^{a}{ }_{\mu}\right\}$ alternative to metric tensor fields $g_{\mu \nu}$. The associated metric space can be constructed from the vierbein fields $g_{\mu \nu}=\eta_{a b} h^{a}{ }_{\mu} h^{b}{ }_{\nu}$ with $\eta_{a b}=$ $\operatorname{diag}(1,-1,-1,-1)$ being the Minkowskian metric of the tangent space; thus, the Levi-Civita symmetric connection $\stackrel{\circ}{\Gamma}_{\mu \nu}^{\alpha}$ is constructed from the metric and its first derivative [77]. Within TEGR, it is possible to build a nonsymmetric connection, Weitzenböck, $\Gamma_{\mu \nu}^{\alpha}=h_{\mu}^{a} \partial_{\nu} h_{a}{ }^{\alpha}=-h_{a}^{\alpha} \partial_{\nu} h_{\mu}^{a}$. The tetrad 4-space is depicted as a pair $\left(M, h_{a}\right)$, where $M$ is a 4 -dimensional smooth manifold and $h_{a}(a=0, \ldots, 3)$ are 4-linearly independent vector fields defined globally on $M$. The tetrad space has a main merit that is the vanishing of the vierbein's covariant derivative; that is, $\nabla_{\nu} h_{\mu}^{a} \equiv 0$, where the covariant derivative, $\nabla$, is regarding the nonsymmetric Weitzenböck connection. Therefore, the vanishing of the vierbein's covariant derivative recognizes autoparallelism or absolute parallelism condition. Actually, the $\nabla$ operator is not invariant under local Lorentz transformations (LLT). In this respect, the symmetric metric (10 degrees of freedom) cannot guess one set of vierbein fields; then, the extra degrees of freedom need to be determined so as one physical frame is used. The vector fields $h_{a}$ are called the parallelization vector fields. Because of the absolute parallelism condition, it can be shown that the metricity condition is satisfied. The Weitzenböck connection is curvatureless while it has a nonvanishing torsion tensor $T$ given as

$$
T_{\mu \nu}^{\lambda}:=h_{a}^{\lambda}\left(\partial_{\mu} h_{\nu}^{a}-\partial_{\nu} h_{\mu}^{a}\right)
$$

and contortion tensor $K$ as

$$
K_{\alpha}^{\mu \nu}=-\frac{1}{2}\left(T_{\alpha}^{\mu \nu}-T_{\alpha}^{\nu \mu}-T_{\alpha}^{\mu \nu}\right) .
$$

The teleparallel torsion scalar which reproduces the TEGR theory is given by

$$
T:=T_{\mu \nu}^{\alpha} S_{\alpha}^{\mu \nu}
$$

where the tensor $S$ of type $(2,1)$ is defined as

$$
S_{\alpha}^{\mu \nu}:=\frac{1}{2}\left(K_{\alpha}^{\mu \nu}+\delta_{\alpha}^{\mu} T_{\beta}^{\beta \nu}-\delta_{\alpha}^{\nu} T_{\beta}^{\beta \mu}\right)
$$

which is skew symmetric in the last two indices. Similar to the $f(R)$ theory, one can define the action of $f(T)$ theory as

$$
\begin{array}{r}
\mathscr{L}\left(h_{\mu}^{a}, \Phi_{A}\right)=\int d^{4} x h\left[\frac{1}{16 \pi} f(T)+\mathscr{L}_{\text {Matter }}\left(\Phi_{A}\right)\right], \\
\text { where } h=\sqrt{-g}=\operatorname{det}\left(h^{a}{ }_{\mu}\right),
\end{array}
$$

and we have assumed the units in which $G=c=1$ and $\Phi_{A}$ are the matter fields. Considering the action (5) as a function of the fields $h_{\mu}^{a}$ and putting the variation of the function regarding the field $h^{a}{ }_{\mu}$ to be vanishing, one can obtain the following equations of motion $[36,71]$ :

$$
\begin{aligned}
S_{\mu}{ }^{\rho \nu} T_{, \rho} & f(T)_{T T} \\
& +\left[h^{-1} h^{a}{ }_{\mu} \partial_{\rho}\left(h h_{a}{ }^{\alpha} S_{\alpha}{ }^{\rho \nu}\right)-T_{\lambda \mu}^{\alpha} S_{\alpha}{ }^{\nu \lambda}\right] f(T)_{T} \\
& -\frac{1}{4} \delta_{\mu}^{\nu} f(T)=-4 \pi \mathfrak{I}_{\mu}{ }^{\nu},
\end{aligned}
$$

where $T_{, \rho}=\partial T / \partial x^{\rho}, f(T)_{T}=\partial f(T) / \partial T, f(T)_{T T}=\partial^{2} f(T) /$ $\partial T^{2}$, and $\mathfrak{I}_{\mu}{ }^{\nu}$ denotes the energy-momentum tensor of the anisotropic fluid which is defined as

$$
\mathfrak{T}_{\mu}{ }^{\nu}=\left(\rho+p_{t}\right) u_{\mu} u^{\nu}-p_{t} \delta_{\mu}^{\nu}+\left(p_{r}-p_{t}\right) \eta_{\mu} \eta^{\nu}
$$

with $p_{r}$ representing the radial pressure, $p_{t}$ representing the tangential pressure, and

$$
\begin{aligned}
& u_{\mu} u^{\mu}=-\eta_{\mu} \eta^{\mu}=1 \\
& u^{\mu} \eta_{\mu}=0
\end{aligned}
$$

Equations (6) are the field equations of $f(T)$ gravitational theory.

\section{Nonvacuum Spherically Symmetric Solutions in Higher-Order Torsion Scalar Theories}

In this section, we are going to apply two, nondiagonal, different tetrad fields having spherical symmetry to the field equations (6). 
3.1. First Tetrad. The equation of motion of GR supplies rich field to use symmetries which link geometry and matter in a natural way. Collineations are symmetries which come from either geometrical viewpoint or physical relevant quantities. The importance of collineations is the CKV which provides more information of the construction of the spacetime geometry. In the language of mathematics, CKV are the motions in which the metric tensor of a spacetime becomes invariant up to a scale factor. Also, the employment of the CKV simplifies the generation of exact solutions to the equations of motions of GR. This is done by reducing the complicated nonlinear partial differential equations of GR to simple ordinary differential equations. The CKV is defined as

$$
\mathbf{L}_{\zeta} g_{i j}=\zeta_{i ; j}+\zeta_{j ; i}=\psi g_{i j}
$$

with $\mathbf{L}$ being the Lie derivative operator of the metric tensor and $\psi$ the conformal factor. One can assume the vector $\zeta$ which creates the conformal symmetry and makes the metric conformally mapped onto itself through $\zeta$. One must note that $\zeta$ and $\psi$ are not necessarily static even supposing a static metric $[78,79]$. In addition, one must be careful about the following:

(i) If $\psi=0$, then (9) leads to a Killing vector.

(ii) If $\psi=$ constant, then (9) leads to homothetic vector.

(iii) If $\psi=\psi(x, t)$, then (9) yields conformal vectors. Furthermore, if $\psi=0$, then the spacetime becomes asymptotically flat and one has a null Weyl tensor. Thus, to have more understanding of the spacetime geometry, one must take into account the CKV. Essentially, the Lie derivative operator $\mathbf{L}$ shows the interior gravitational field of a stellar configuration related to the vector field $\zeta$.

The first tetrad field having a stationary and spherical symmetry with local Lorentz transformations has the following form [80]:

$$
\left(h_{\mu}^{i}\right)=\left(\begin{array}{cccc}
\frac{\mathscr{F}_{1}(r)}{\mathscr{F}_{2}(r)} & \mathscr{F}_{2}(r) & 0 & 0 \\
\sin \theta \cos \phi & \mathscr{F}_{1}(r) \sin \theta \cos \phi & r \cos \theta \cos \phi & -r \sin \theta \sin \phi \\
\sin \theta \sin \phi & \mathscr{F}_{1}(r) \sin \theta \sin \phi & r \cos \theta \sin \phi & r \sin \theta \cos \phi \\
\cos \theta & \mathscr{F}_{1}(r) \cos \theta & -r \sin \theta &
\end{array}\right)
$$

where $\mathscr{F}_{1}(r)$ and $\mathscr{F}_{2}(r)$ are two unknown functions of the radial coordinate, $r$.

The associated metric of (10) takes the following form:

$$
\begin{array}{r}
d s^{2}=-\frac{\mathscr{F}_{1}{ }^{2}-\mathscr{F}_{2}{ }^{2}}{\mathscr{F}_{2}{ }^{2}} d t^{2}+\left(\mathscr{F}_{1}{ }^{2}-\mathscr{F}_{2}{ }^{2}\right) d r^{2}+d \Omega, \\
d \Omega=r^{2}\left(d \theta^{2}+\sin ^{2} \theta d \phi^{2}\right),
\end{array}
$$

which is a static spherically symmetric spacetime that admits one parameter group of conformal motion. Equation (11) is conformally mapped onto itself along $\zeta$. Therefore, (9) leads to

$$
\begin{aligned}
& 2\left[\mathscr{F}_{1}^{\prime} \mathscr{F}_{1} \mathscr{F}_{2}-\mathscr{F}_{1}{ }_{1}^{2} \mathscr{F}_{2}^{\prime}\right] \zeta^{1}=\psi(r)\left[\mathscr{F}_{1}{ }^{2} \mathscr{F}_{2}-\mathscr{F}_{2}{ }^{3}\right], \\
& \zeta^{0}=c \\
& \zeta^{1}=\frac{\psi(r) r}{2}
\end{aligned}
$$

$$
\begin{aligned}
2 \zeta^{1} & {\left[\mathscr{F}_{1} \mathscr{F}_{1}^{\prime}-\mathscr{F}_{2} \mathscr{F}_{2}^{\prime}\right]+2 \zeta^{\prime 1}\left[\mathscr{F}_{1}{ }^{2}-\mathscr{F}_{2}{ }^{2}\right] } \\
& =\psi(r)\left[\mathscr{F}_{1}{ }^{2}-\mathscr{F}_{2}{ }^{2}\right]
\end{aligned}
$$

where 0 and 1 refer to the temporal and spatial coordinates $r$ and $t$, respectively. The above set of equations lead to

$$
\begin{aligned}
\mathscr{F}_{1} & =\frac{\sqrt{1+c_{0}{ }^{2} r^{2}} \mathscr{F}_{3}}{c_{0} r}, \\
\mathscr{F}_{2} & =\frac{\mathscr{F}_{3}}{r c_{0}}, \\
\zeta^{i} & =c_{1} \delta_{0}^{i}+\frac{\psi(r) r}{2} \delta^{i}, \\
\mathscr{F}_{1} & \neq \mathscr{F}_{2}, \\
\mathscr{F}_{3} & =\frac{c_{2}}{\psi(r)},
\end{aligned}
$$

with $c, c_{0}, c_{1}$, and $c_{2}$ being constants of integration. 
Using (13), tetrad (10) is rewritten as

$$
\left(h_{\mu}^{i}\right)=\left(\begin{array}{cccc}
\sqrt{1+c_{0}{ }^{2} r^{2}} & \frac{\mathscr{F}_{3}}{r c_{0}} & 0 & 0 \\
\sin \theta \cos \phi & \frac{\mathscr{F}_{3} \sqrt{1+c_{0}{ }^{2} r^{2}} \sin \theta \cos \phi}{r c_{0}} & r \cos \theta \cos \phi & -r \sin \theta \sin \phi \\
\sin \theta \sin \phi & \frac{\mathscr{F}_{3} \sqrt{1+c_{0}{ }^{2} r^{2}} \sin \theta \sin \phi}{r c_{0}} & r \cos \theta \sin \phi & r \sin \theta \cos \phi \\
\cos \theta & \frac{\mathscr{F}_{3} \sqrt{1+c_{0}{ }^{2} r^{2}} \cos \theta}{r c_{0}} & -r \sin \theta & 0
\end{array}\right),
$$

Tetrad field (14) has the following associated metric:

$d s^{2}=-c_{0}{ }^{2} r^{2} d t^{2}+\mathscr{F}_{3}{ }^{2} d r^{2}+d \Omega$,

$$
d \Omega=r^{2}\left(d \theta^{2}+\sin ^{2} \theta d \phi^{2}\right) .
$$

Using (14) in (3), we get the scalar torsion in the following form:

$$
=2 \frac{2\left(1+2 c_{0}^{2} r^{2}\right) \mathscr{F}_{3}(r)-r c_{0} \sqrt{1+c_{0}^{2} r^{2}}\left(3+\mathscr{F}_{3}^{2}(r)\right)}{c_{0} r^{3} \sqrt{1+c_{0}{ }^{2} r^{2}} \mathscr{F}_{3}^{2}(r)} .
$$

Using (16) and (14) in the field equations (6), we get the following nonvanishing components:

$$
\begin{aligned}
& 4 \pi \mathscr{T}_{0}{ }^{0}=4 \pi \rho=-\frac{\sqrt{1+c_{0}{ }^{2} r^{2}} \mathscr{F}_{3}-r c_{0}}{r^{2} c_{0} \mathscr{F}_{3}^{2}} T^{\prime} f_{T T} \\
& +\frac{r c_{0} \sqrt{1+c_{0}{ }^{2} r^{2}}\left(2 \mathscr{F}_{3}-r \mathscr{F}_{3}^{\prime}\right)-\mathscr{F}_{3}^{2}\left(1+2 c_{0}{ }^{2} r^{2}\right)}{r^{3} c_{0} \sqrt{1+c_{0}{ }^{2} r^{2}} \mathscr{F}_{1}{ }^{3}} f_{T}+\frac{f}{4}, \\
& 4 \pi \mathscr{T}_{1}{ }^{0}=-\frac{T^{\prime} f_{T T}}{r^{3} c_{0}{ }^{2}}
\end{aligned}
$$

$$
\begin{aligned}
& -4 \pi \mathscr{T}_{1}{ }^{1}=4 \pi p_{r}=-\frac{\mathscr{F}_{3}\left(1+2 r^{2} c_{0}{ }^{2}\right)-3 r c_{0} \sqrt{1+c_{0}{ }^{2} r^{2}}}{r^{3} c_{0} \mathscr{F}_{1}{ }^{2} \sqrt{1+c_{0}{ }^{2} r^{2}}} f_{T} \\
& +\frac{f}{4}, \\
& -4 \pi \mathscr{T}_{2}{ }^{2}=-4 \pi \mathscr{T}_{3}{ }^{3}=4 \pi p_{t}=-\frac{\sqrt{1+c_{0}{ }^{2} r^{2}} \mathscr{F}_{3}-2 r c_{0}}{2 r^{2} c_{0} \mathscr{F}_{3}{ }^{2}} T^{\prime} f_{T T} \\
& +\frac{r c_{0} \sqrt{1+c_{0}{ }^{2} r^{2}}\left(\mathscr{F}_{3}{ }^{3}+4 \mathscr{F}_{3}-2 r \mathscr{F}_{3}^{\prime}\right)-2 \mathscr{F}_{3}{ }^{2}\left(1+2 c_{0}{ }^{2} r^{2}\right)}{2 r^{3} c_{0} \sqrt{1+c_{0}{ }^{2} r^{2} \mathscr{F}_{3}{ }^{3}}} \\
& \cdot f_{T}+\frac{f}{4} .
\end{aligned}
$$

Second equation of (17) leads to $f_{T T}=0$, or $T=$ constant. The case $T=$ constant gives a constant function and this is out of the scope of the present study. Therefore, we are searching for solutions that make constraint on the form of $f(T)$ have the form:

$$
\begin{aligned}
f(T) & =T, \Longrightarrow \\
f_{T T} & =0 .
\end{aligned}
$$

Assuming the isotropic condition

$$
p_{r}=p_{t}=p
$$

and using (19) in (17), we get

$$
\begin{aligned}
\mathscr{F}_{3}(r) & =\frac{2}{\sqrt{2+4 r^{2} c_{3}}}, \\
T & =-\frac{6 c_{3} c_{0} r^{3} \sqrt{1+r^{2} c_{0}^{2}}+5 r c_{0} \sqrt{1+r^{2} c_{0}^{2}}-2\left(1+2 c_{0}{ }^{2} r^{2}\right) \sqrt{2+4 r^{2} c_{3}}}{r^{3} c_{0} \sqrt{1+r^{2} c_{0}^{2}}},
\end{aligned}
$$




$$
\begin{aligned}
& 16 \pi \rho=\frac{6 c_{3} r^{2}-1}{r^{2}}, \\
& 16 \pi p=\frac{6 c_{3} r^{2}+1}{r^{2}}, \\
& \psi(r)=\frac{c_{2} \sqrt{2+4 r^{2} c_{3}}}{2} .
\end{aligned}
$$

The sound velocity $v_{s}{ }^{2}$ is defined as $v_{s}{ }^{2}:=d p / d \rho$. Using (20), we get the sound velocity in the following form:

$$
v_{s}^{2}=-1
$$

3.2. Second Tetrad. The second tetrad space having a stationary and spherical symmetry takes the following form [73]:

$$
\begin{aligned}
& \left(h_{\mu}^{i}\right) \\
& =\left(\begin{array}{cccc}
\mathscr{F}_{4}(r) & 0 & 0 & 0 \\
0 & \mathscr{F}_{5}(r) \sin \theta \cos \phi & r \cos \theta \cos \phi & -r \sin \theta \sin \phi \\
0 & \mathscr{F}_{5}(r) \sin \theta \sin \phi & r \cos \theta \sin \phi & r \sin \theta \cos \phi \\
0 & \mathscr{F}_{5}(r) \cos \theta & -r \sin \theta & 0
\end{array}\right),
\end{aligned}
$$

where $\mathscr{F}_{4}(r)$ and $\mathscr{F}_{5}(r)$ are two unknown functions of the radial coordinate, $r$. Using the same procedure applied to tetrad (10), we get the following equations of CKV of tetrad (22):

$$
\begin{aligned}
2 \mathscr{F}_{4}^{\prime} \xi^{1} & =\psi(r) \mathscr{F}_{4}, \\
\xi^{0} & =c, \\
\xi^{1} & =\frac{\psi(r) r}{2}, \\
2 \xi^{1} \mathscr{F}_{5}^{\prime}+2 \xi_{, 1}^{1} \mathscr{F}_{5} & =\psi(r) \mathscr{F}_{5} .
\end{aligned}
$$

The above set of equations imply

$$
\begin{aligned}
\mathscr{F}_{4} & =c_{4} r, \\
\mathscr{F}_{5} & =\frac{c_{5}}{\psi(r)}, \\
\xi^{i} & =c_{6} \delta^{i}{ }_{0}+\frac{\psi(r) r}{2} \delta_{1}^{i},
\end{aligned}
$$

where $c_{4}, c_{5}$, and $c_{6}$ are constants of integration.

Using (24), tetrad (22) can be rewritten as

$$
\begin{aligned}
& \left(h_{\mu}^{i}\right) \\
& =\left(\begin{array}{cccc}
c_{4} r & 0 & 0 & 0 \\
0 & \mathscr{F}_{5} \sin \theta \cos \phi & r \cos \theta \cos \phi & -r \sin \theta \sin \phi \\
0 & \mathscr{F}_{5} \sin \theta \sin \phi & r \cos \theta \sin \phi & r \sin \theta \cos \phi \\
0 & \mathscr{F}_{5} \cos \theta & -r \sin \theta & 0
\end{array}\right) .
\end{aligned}
$$

Using (25), the torsion scalar (3) takes the following form:

$$
T=\frac{2\left(3-4 \mathscr{F}_{5}+\mathscr{F}_{5}^{2}\right)}{r^{2} \mathscr{F}_{5}{ }^{2}} .
$$

Inserting (26) and the components of the tensors $S_{\rho}^{\nu \mu}$ and $T_{\rho}^{\nu \mu}$ in the field equations (6), we obtain

$$
\begin{aligned}
4 \pi \mathscr{T}_{0}{ }^{0}= & 4 \pi \rho \\
= & \frac{\left(1-\mathscr{F}_{5}\right)}{r \mathscr{F}_{5}{ }^{2}} T^{\prime} f_{T T}-\frac{2 \mathscr{F}_{5}{ }^{2}-2 \mathscr{F}_{5}+r \mathscr{F}_{5}^{\prime}}{r^{2} \mathscr{F}_{5}{ }^{3}} f_{T} \\
& +\frac{f}{4}, \\
-4 \pi \mathscr{T}_{1}{ }^{1}= & 4 \pi p_{r}=\frac{3-2 \mathscr{F}_{5}}{r^{2} \mathscr{F}_{5}{ }^{2}} f_{T}+\frac{f}{4}, \\
-8 \pi \mathscr{T}_{2}{ }^{2}= & -8 \pi \mathscr{T}_{3}{ }^{3}=4 \pi p_{t} \\
= & \frac{\left(2-\mathscr{F}_{5}\right)}{2 r \mathscr{F}_{5}{ }^{2}} T^{\prime} f_{T T} \\
& -\frac{2 \mathscr{F}_{5}{ }^{2}-2 \mathscr{F}_{5}+r \mathscr{F}_{5}^{\prime}-\mathscr{F}_{5}{ }^{3}}{2 r^{2} \mathscr{F}_{5}{ }^{3}} f_{T}+\frac{f}{4} .
\end{aligned}
$$

The above system cannot be solved without assuming some specific constraint on the form of $f(T)$. Therefore, we are going to use the constraint (18) in (27) and obtain the following:

$$
\begin{aligned}
\mathscr{F}_{5}(r) & =\frac{2}{\sqrt{2+4 r^{2} c_{7}}}, \\
T & =\frac{5 \sqrt{2+4 r^{2} c_{7}}-8-16 r^{2} c_{5}+6 c_{7} \sqrt{2+4 r^{2} c_{7}}}{r^{2} \sqrt{2+4 r^{2} c_{7}}}, \\
16 \pi \rho & =\frac{9+18 r^{2} c_{7}-8 \sqrt{2+4 r^{2} c_{7}}}{r^{2}},
\end{aligned}
$$




$$
\begin{aligned}
& 16 \pi p=\frac{11+18 r^{2} c_{7}-8 \sqrt{2+4 r^{2} c_{7}}}{r^{2}}, \\
& \psi(r)=\frac{c_{5} \sqrt{2+4 r^{2} c_{7}}}{2} .
\end{aligned}
$$

Using (28), the sound velocity $v_{s}^{2}$ takes the following form:

$$
\begin{aligned}
& \frac{d p}{d \rho} \\
& =\frac{16+32 r^{4} c_{7}^{2}+48 r^{2} c_{7}-22 r^{2} c_{7} \sqrt{2+4 r^{2} c_{7}}-11 \sqrt{2+4 r^{2} c_{7}}}{\left(8 c_{7} \sqrt{2+4 r^{2} c_{7}}-18 r^{2} c_{7}-9+8 \sqrt{2+4 r^{2} c_{7}}\right) \sqrt{2+4 r^{2} c_{7}}} .
\end{aligned}
$$

\section{Physics Relevant to the Models}

4.1. Energy Conditions. Energy conditions are essential tools to understand various cosmological geometries and some general results related to the strong gravitational fields. These tools are three forms of energy conditions, the strong energy (SEC), null energy (NEC), and weak energy conditions (WEC) [81-83]. Such conditions have the following inequalities:

$$
\begin{array}{cl}
\text { NEC: } & \rho+p_{r} \geq 0, \\
& \rho+p_{t} \geq 0, \\
\text { SEC: } & \rho+p_{r} \geq 0, \\
& \rho+p_{r}+2 p_{t} \geq 0, \\
\text { WEC: } & \rho \geq 0, \\
& \rho+p_{r} \geq 0, \\
& \rho+p_{t} \geq 0 .
\end{array}
$$

It is interesting to remember that the breaking of the conditions leads to the existence of the ghost instabilities.

4.2. Energy Conditions of Smooth Transition Models. Let us apply the above procedure of the energy conditions given by (30) to the derived solutions given in the previous section. Equations (28) show that density has a positive value and $\rho+$ $p \geq 0, \rho+3 p \geq 0$ are satisfied with constant $c_{3}>0$ for the first model and $c_{7}>0$ for the second model as shown in Figures 1 and 2. This means that NEC, SEC, and WEC are satisfied for the above two models. Also, it is interesting to note that the density and pressure of both solutions do not depend on the constants $c_{0}$ and $c_{4}$.

4.3. Stability Problem. To study the stability issue of the above two models, we use the cracking mechanism [84] in which the squares of the sound speed should be in the range $[0,1]$; that is, $0 \leq v_{s}{ }^{2} \leq 1$. Figure 3(a) does not show the positivity criterion; that is, $v_{s}{ }^{2} \leq 0$. However, Figure $3(\mathrm{~b})$ satisfies the criterion of stability; that is, $v_{s}{ }^{2} \geq 0$ within the matter distribution provided any value of the constant $c_{7}$, in Figure 3(b), and therefore second model maintains stability.

4.4. Nature of the Star. To understand the nature of the star, we draw a plot to indicate the radius of the stellar model for the second model. It can be seen by the clear cut on $r$-axis which is turned out to be approximately $1 \mathrm{~km}$ (Figure 4). This value is very small and shows a compact star with ultracompactness. A tally of this value with the already available data set immediately reveals that the star is nothing but either a quark/strange star $[85,86]$ or a brown dwarf star of type F5. This value of $R \sim 1 \mathrm{~km}$ makes us detect the surface density of the stellar system. As $r$ approaches zero, density approaches $\infty$ and, therefore, the central density is out of the scope of this study. However, we can guess the surface density of the star by plugging the values of the Newtonian constant, $G$, and the speed of light, $c$, in the expression of density which gives the numerical value as $13 \mathrm{gm} / \mathrm{cm}^{3}$. This is a normal energy density in which the radius $R=1 \mathrm{~km}$ is very small. This indicates that the second model under $f(T)$ gravity represents an ultracompact star [87-89]. The first model is not a physical one because of the fact that, to find the cutting of the pressure with the $r$-axis, the constant $c_{3}$ will have a negative value which makes a contradiction with the energy conditions.

4.5. TOV Equation. The TOV equation for a metric in the form

$$
d s^{2}=-e^{\nu(r)} d t^{2}+e^{\lambda(r)} d r^{2}+r^{2}\left(d \theta^{2}+\sin ^{2} \theta d \phi^{2}\right)
$$

can be written in the following form [85]:

$$
\begin{aligned}
& -\frac{M_{G(r)}\left(\rho+p_{r}\right) e^{(\lambda(r)-\gamma(r)) / 2}}{r^{2}}-\frac{d p_{r}}{d r}+\frac{2\left(p_{t}-p_{r}\right)}{r} \\
& \quad=0,
\end{aligned}
$$

where $M_{G(r)}$ is the gravitational mass in the sphere of radius $r$ which is given by

$$
M_{G(r)}=\frac{r^{2} \nu^{\prime} e^{(\lambda(r)-\nu(r)) / 2}}{2} .
$$

Using (33) in (32), we obtain in the isotropic case

$$
-\frac{v^{\prime}\left(\rho+p_{r}\right)}{2}-\frac{d p_{r}}{d r}=0 .
$$




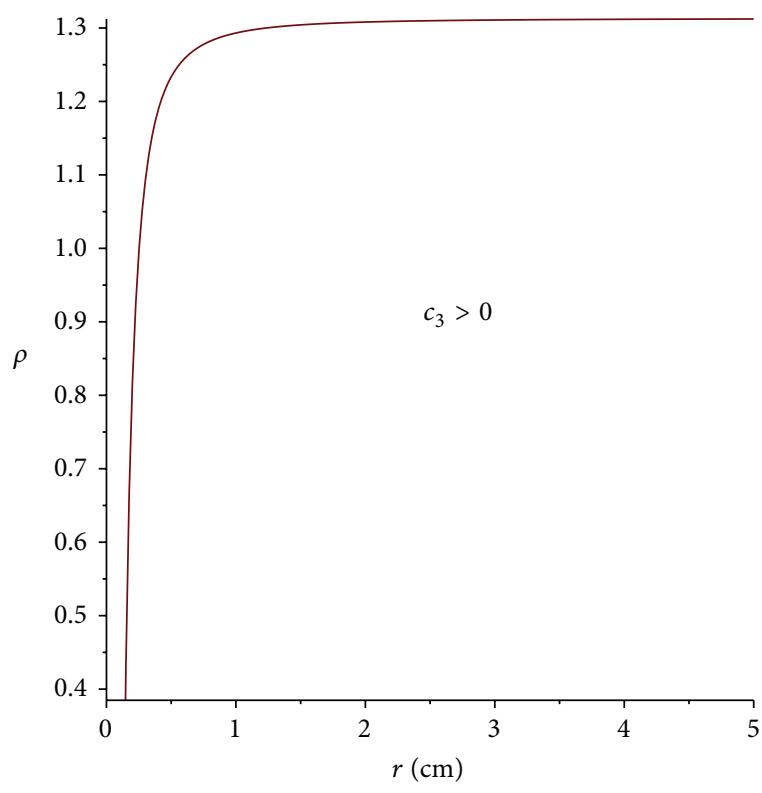

(a) $\rho$

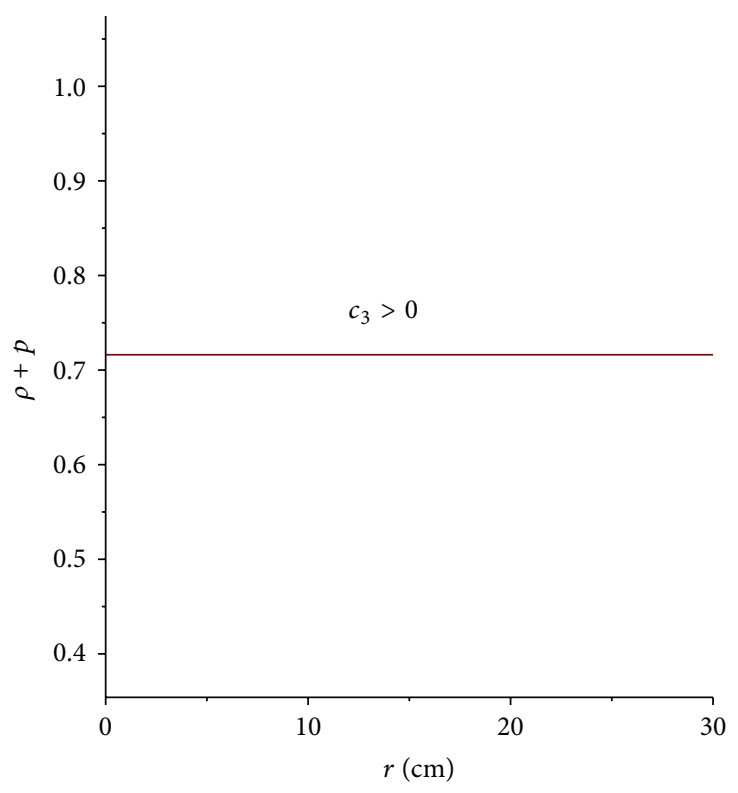

(b) $\rho+p$

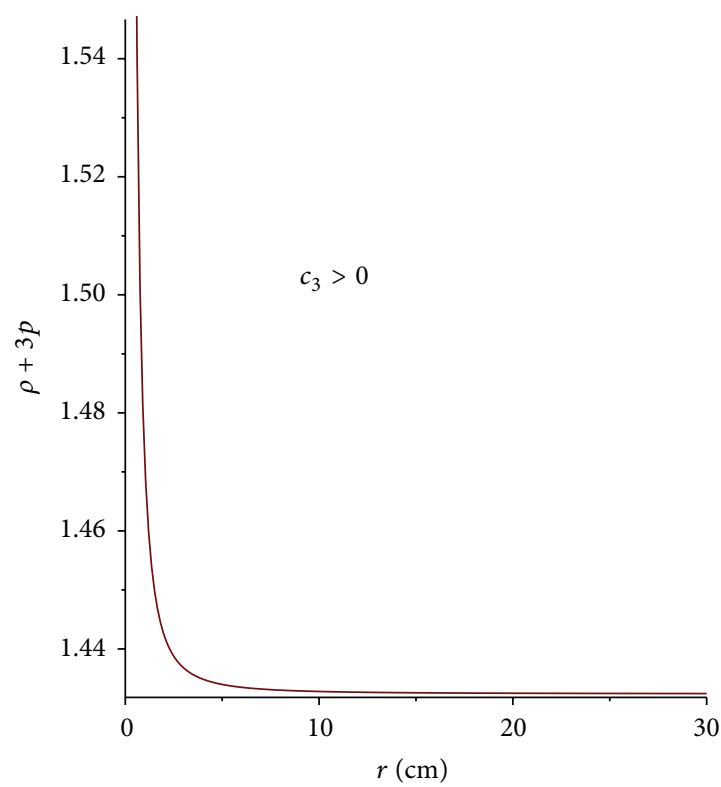

(c) $\rho+3 p$

FIGURE 1: Energy conditions of the first model: the constant $c_{3}$ assumes a positive value so that the density is positive and also the pressure.

Equation (34) demonstrates the equilibrium of the stellar configuration under the action of different forces, that is, gravitational force $F_{g}$ and hydrostatic force $F_{h}$; therefore, as an equilibrium condition, we write (34) in the following form:

$$
F_{g}+F_{h}=0
$$

where

$$
\begin{aligned}
& F_{g}=-\frac{\nu^{\prime}\left(\rho+p_{r}\right)}{2}, \\
& F_{h}=-\frac{d p_{r}}{d r} .
\end{aligned}
$$

Using (14), (20), (25), and (28), we plot the feature of TOV equation for the above two models in Figure 5.

\section{Conclusion and Discussion}

In this study, we have used two nondiagonal different tetrad fields having spherical symmetry and reproduce the same associated metric. These tetrads are connected by local Lorentz transformation. We have used the CKV mechanism to reduce the highly nonlinear partial differential equations. We have applied the field equations of $f(T)$ to the first 


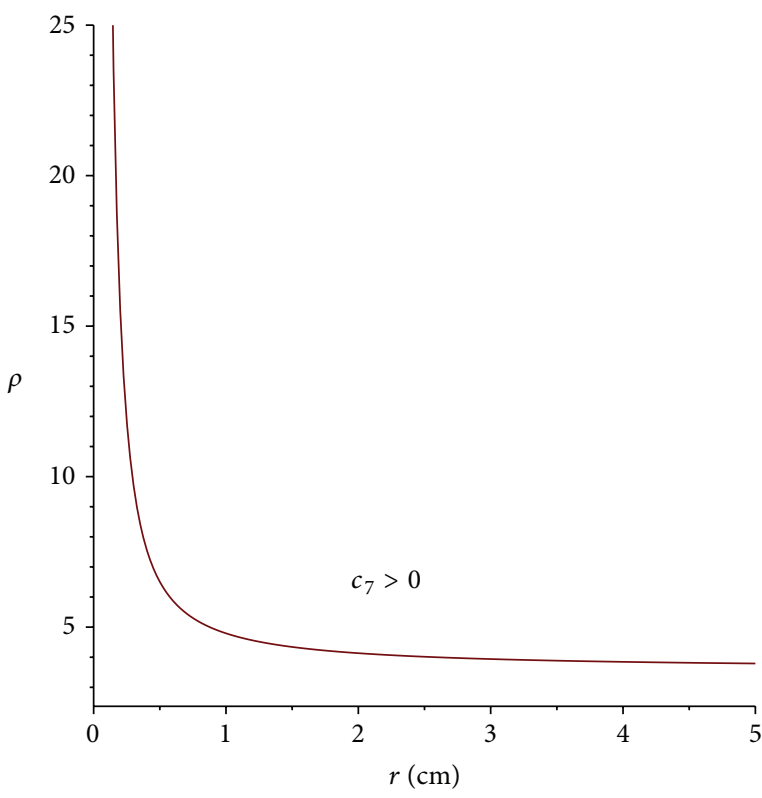

(a) $\rho$

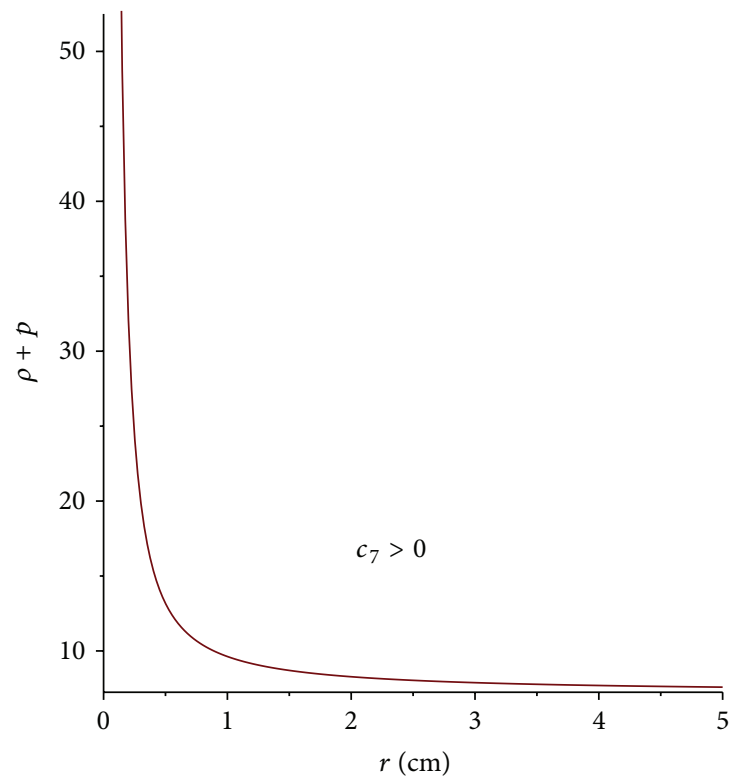

(b) $\rho+p$

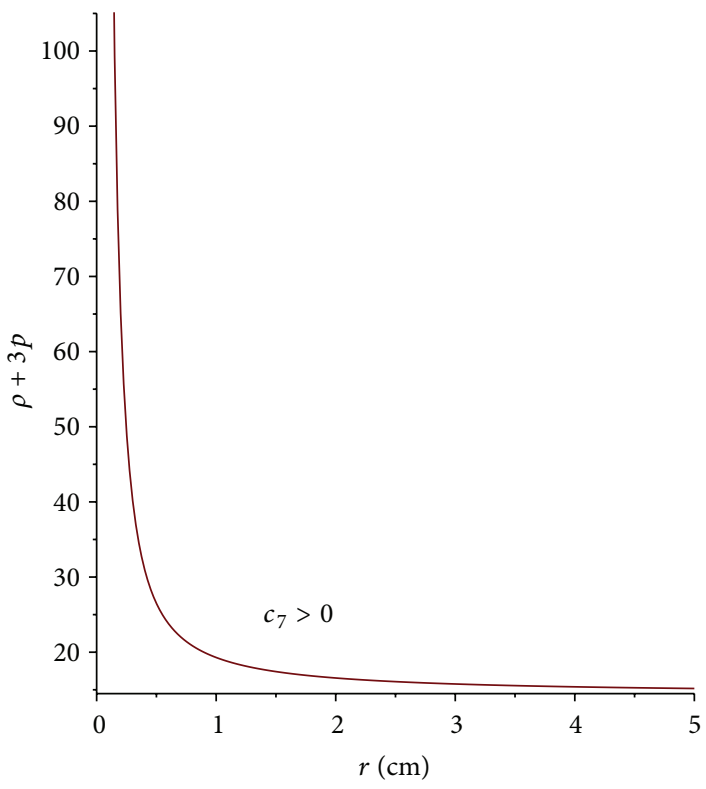

(c) $\rho+3 p$

FIGURE 2: Energy conditions of the second model: the constant $c_{7}$ assumes a positive value so that the density is positive and also the pressure.

tetrad and have obtained anisotropic system that consists of four nonlinear differential equations. One of these deferential equations put a constraint on the form of $f(T)$. This constraint makes the form of $f(T)$ be $f(T)=T$. Using this form and the isotropic condition, that is, $p_{r}=p_{t}$, we get an isotropic solution.

For the second tetrad, we have obtained anisotropic system that consists of three nonlinear differential equations. We cannot solve this system without any constraints on the form of $f(T)$. Using the constraint of $f(T)$ applied to the first tetrad, that is, $f(T)=T$, and the condition of isotropy, we get another solution.

We have studied the physics relevant to each solution and have shown that the first and second tetrads satisfied the energy conditions provided that the two constants of integration involved in these solutions are positive. We have shown that the first tetrad is not stable one because the sound speed is negative; that is, $d p / d \rho<0$ [84]. However, the second model has confirmed stable manner and has shown a dynamical behavior. We have indicated that the first tetrad 


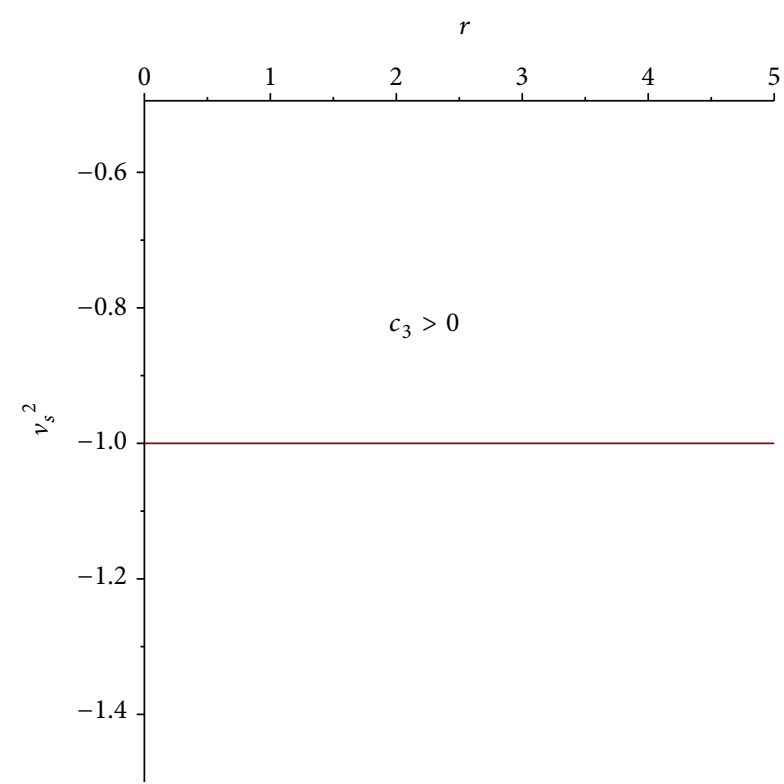

(a) $v_{s}^{2}<0$

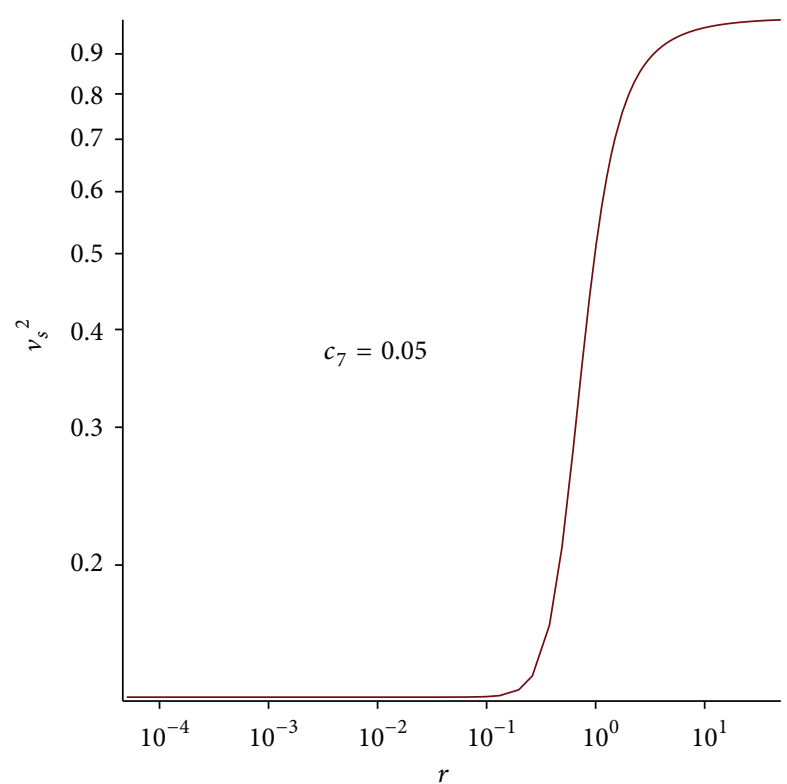

(b) $v_{s}^{2} \geq 0$

FIGURE 3: (a) shows that the first model does not have stability because the sound velocity $v_{s}^{2} \notin[0,1]$ as is required while (b) shows a stability behavior.

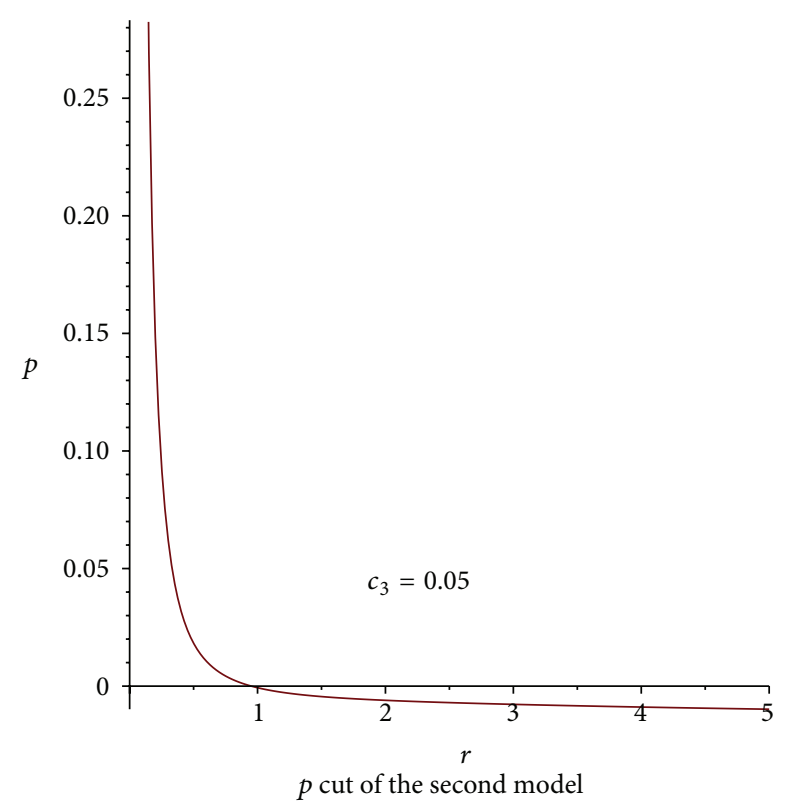

FIGURE 4: Radius of the star is given where the pressure $p$ cuts $r$-axis.

is not suitable to construct a stellar model because the radius has an imaginary quantity. In the meanwhile, the second model has illustrated a stellar model that has a radius about $1 \mathrm{~km}$ and the value of density is not intensive enough on the surface. Finally, we have shown that the plots for the generalized TOV equation show that static equilibrium has been attained by different forces, that is, gravitational force $F_{g}$ and hydrostatic force $F_{h}$. Figure 5(b) shows that the second model has a tendency toward equilibrium while the first one did not show such equilibrium.

\section{Conflict of Interests}

The author declares that there is no conflict of interests regarding the publication of this paper. 


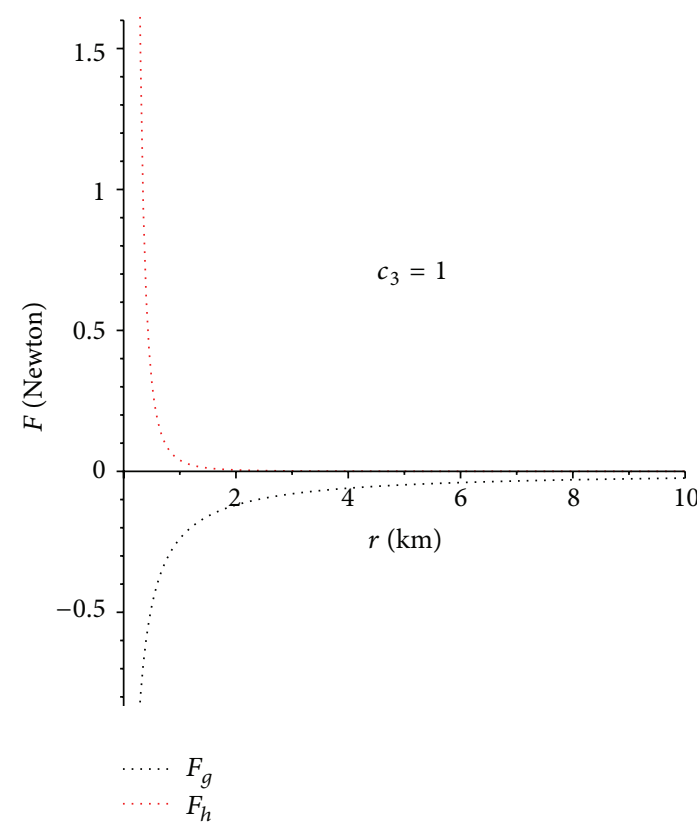

(a) TOV of the first model

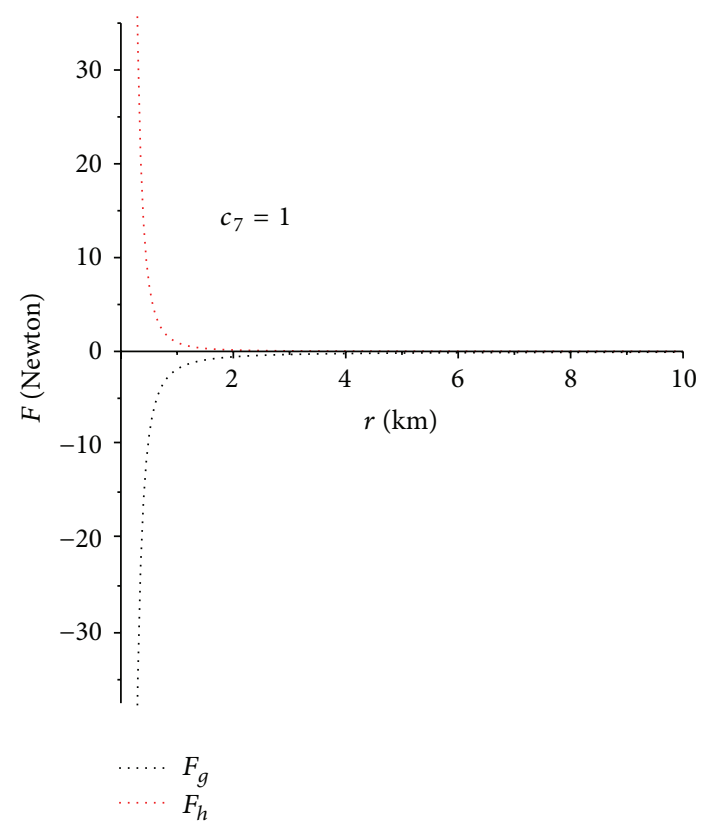

(b) TOV of the second model

FIGURE 5: The two different forces, that is, gravitational force $F_{g}$ and hydrostatic force $F_{h}$, are plotted against $r$ (km) for the two models.

\section{Acknowledgment}

This work is partially supported by the Egyptian Ministry of Scientific Research under Project no. 24-2-12.

\section{References}

[1] R. Ferraro and F. Fiorini, "Modified teleparallel gravity: inflation without an inflaton," Physical Review D, vol. 75, no. 8, Article ID 084031, 5 pages, 2007.

[2] E. V. Linder, "Einstein's other gravity and the acceleration of the universe," Physical Review D, vol. 81, no. 12, Article ID 127301, 2010.

[3] P. Wu and H. W. Yu, " $f(T)$ models with phantom divide line crossing," The European Physical Journal C, vol. 71, article 1552, 2011.

[4] K. Bamba, C.-Q. Geng, C.-C. Lee, and L.-W. Luo, "Equation of state for dark energy in $f(T)$ gravity," Journal of Cosmology and Astroparticle Physics, vol. 2011, no. 1, article 021, 2011.

[5] K. Bamba, S. D. Odintsov, and D. Sáez-Gómez, "Conformal symmetry and accelerating cosmology in teleparallel gravity," Physical Review D, vol. 88, no. 8, 2013.

[6] J. B. Dent, S. Dutta, and E. N. Saridakis, “ $f(T)$ gravity mimicking dynamical dark energy. Background and perturbation analysis," Journal of Cosmology and Astroparticle Physics, vol. 2011, no. 1, article 009, 2011.

[7] K. Bamba, R. Myrzakulov, S. Nojiri, and S. D. Odintsov, "Reconstruction of $f(T)$ gravity: rip cosmology, finite-time future singularities, and thermodynamics," Physical Review D, vol. 85, Article ID 104036, 2012.

[8] A. Aviles, A. Bravetti, S. Capozziello, and O. Luongo, "Cosmographic reconstruction of $f(\mathscr{T})$ cosmology," Physical Review D, vol. 87, no. 6, Article ID 064025, 2013.
[9] M. Jamil, D. Momeni, and R. Myrzakulov, "Stability of a nonminimally conformally coupled scalar field in $F(T)$ cosmology," The European Physical Journal C, vol. 72, article 2075, 2012.

[10] M. Sharif and S. Rani, "Generalized teleparallel gravity via some scalar field dark energy models," Astrophysics and Space Science, vol. 345, no. 1, pp. 217-223, 2013.

[11] R. Ferraro and F. Fiorini, "Non-trivial frames for $f(T)$ theories of gravity and beyond," Physics Letters B, vol. 702, no. 1, pp. 7580, 2011.

[12] F. Fiorini and R. Ferraro, "Cosmological frames for theories with absolute parallelism," International Journal of Modern Physics: Conference Series, vol. 3, pp. 227-237, 2011.

[13] P. Wu and H. W. Yu, "Observational constraints on $f(T)$ theory," Physics Letters B, vol. 693, no. 4, pp. 415-420, 2010.

[14] I. G. Salako, M. E. Rodrigues, A. V. Kpadonou, M. J. S. Houndjo, and J. Tossa, " $\Lambda C D M$ model in $f(T)$ gravity: reconstruction, thermodynamics and stability," Journal of Cosmology and Astroparticle Physics, vol. 2013, no. 11, article 060, 2013.

[15] Z. Haghani, T. Harko, H. R. Sepangi, and S. Shahidi, "WeylCartan-Weitzenboeck gravity as a generalization of teleparallel gravity," Journal of Cosmology and Astroparticle Physics, vol. 2012, no. 10, article 061, 2012.

[16] Z. Haghani, T. Harko, H. R. Sepangi, and S. Shahidi, "WeylCartan-Weitzenböck gravity through Lagrange multiplier," Physical Review D-Particles, Fields, Gravitation and Cosmology, vol. 88, no. 4, Article ID 044024, 2013.

[17] T. Shirafuji and G. G. Nashed, "Energy and momentum in the tetrad theory of gravitation," Progress of Theoretical Physics, vol. 98, no. 6, pp. 1355-1370, 1997.

[18] K. Bamba, S. Nojiri, and S. D. Odintsov, "Effective $F(T)$ gravity from the higher-dimensional Kaluza-Klein and RandallSundrum theories," Physics Letters B, vol. 725, no. 4-5, pp. 368371, 2013. 
[19] K. Bamba, J. de Haro, and S. D. Odintsov, "Future singularities and teleparallelism in loop quantum cosmology," Journal of Cosmology and Astroparticle Physics, vol. 2013, no. 02, article 008, 2013.

[20] S. Nojiri and S. D. Odintsov, "Unified cosmic history in modified gravity: from $F(R)$ theory to Lorentz non-invariant models," Physics Reports, vol. 505, no. 2-4, pp. 59-144, 2011.

[21] G. G. Nashed, "Charged axially symmetric solution, energy and angular momentum in tetrad theory of gravitation," International Journal of Modern Physics A, vol. 21, no. 15, pp. 3181-3197, 2006.

[22] S. Nojiri and S. D. Odintsov, "Introduction to modified gravity and gravitational alternative for dark energy," International Journal of Geometric Methods in Modern Physics, vol. 4, no. 1, pp. 115-145, 2007.

[23] S. Capozziello and V. Faraoni, Beyond Einstein Gravity, Springer, Berlin, Germany, 2010.

[24] S. Capozziello and M. De Laurentis, "Extended theories of gravity," Physics Reports, vol. 509, no. 4-5, pp. 167-321, 2011.

[25] A. de la Cruz-Dombriz and D. Sáez-Gómez, "Black holes, cosmological solutions, future singularities, and their thermodynamical properties in modified gravity theories," Entropy, vol. 14, no. 9, pp. 1717-1770, 2012.

[26] S.-H. Chen, J. B. Dent, S. Dutta, and E. N. Saridakis, "Cosmological perturbations in $f(T)$ gravity," Physical Review D, vol. 83, no. 2, Article ID 023508, 2011.

[27] Y.-P. Wu and C.-Q. Geng, "Matter density perturbations in modified teleparallel theories," Journal of High Energy Physics, vol. 2012, no. 11, article 142, 2012.

[28] K. Bamba, S. Capozziello, S. Nojiri, and S. D. Odintsov, "Dark energy cosmology: the equivalent description via different theoretical models and cosmography tests," Astrophysics and Space Science, vol. 342, no. 1, pp. 155-228, 2012.

[29] B. Li, T. P. Sotirious, and J. D. Barrow, " $f(T)$ gravity and local Lorentz invariance," Physical Review D, vol. 83, no. 6, Article ID 064035, 5 pages, 2011.

[30] T. P. Sotirious, B. Li, and J. D. Barrow, "Generalizations of teleparallel gravity and local Lorentz symmetry," Physical Review D, vol. 83, no. 10, Article ID 104030, 2011.

[31] M. Kššárk and E. N. Saridakis, "The covariant formulationof $f(T)$ gravity," http://arxiv.org/abs/1510.08432.

[32] C.-Q. Geng, C.-C. Lee, E. N. Saridakis, and Y.-P. Wu, “'Teleparallel' dark energy," Physics Letters B, vol. 704, no. 5, pp. 384-387, 2011.

[33] C.-Q. Geng, J.-A. Gu, and C.-C. Lee, "Singularity problem in teleparallel dark energy models," Physical Review D, vol. 88, no. 2, Article ID 024030, 2013.

[34] C.-Q. Geng, C.-C. Lee, and E. N. Saridakis, "Observational constraints on teleparallel dark energy," Journal of Cosmology and Astroparticle Physics, vol. 2012, no. 1, article 002, 2012.

[35] Y. C. Ong, K. Izumi, J. M. Nester, and P. Chen, "Problems with propagation and time evolution in $f(T)$ gravity," Physical Review $D$, vol. 88, no. 2, Article ID 024019, 2013.

[36] G. R. Bengochea and R. Ferraro, "Dark torsion as the cosmic speed-up," Physical Review D, vol. 79, no. 12, Article ID 124019, 2009.

[37] C. Xu, E. N. Saridakis, and G. Leon, "Phase-space analysis of teleparallel dark energy," Journal of Cosmology and Astroparticle Physics, vol. 2012, no. 7, article 005, 2012.

[38] Y.-F. Cai, S.-H. Chen, J. B. Dent, S. Dutta, and E. N. Saridakis, "Matter bounce cosmology with the $f(T)$ gravity," Classical and Quantum Gravity, vol. 28, no. 21, Article ID 215011, 2011.
[39] A. Einstein, "Riemann-geometrie mit Aufrechterhaltung des Begriffes des Fern-Parallelismus," Sitzungsberichte der Preussischen Akademie der Wissenschaften, Physikalisch-Mathematische Klasse, pp. 217-221, 1928.

[40] A. Einstein, "Zur Theorie der Räume mit Riemannmetrik und Fernparallelismus," Sitzungsberichte der Preussischen Akademie der Wissenschaften, Physikalisch-Mathematische Klasse, pp. 401402, 1930.

[41] J. Yang, Y.-L. Li, Y. Zhong, and Y. Li, “Thick brane split caused by spacetime torsion," Physical Review D, vol. 85, no. 8, Article ID 084033, 2012.

[42] K. Karami and A. Abdolmaleki, "Generalized second law of thermodynamics in $f(T)$ gravity," Journal of Cosmology and Astroparticle Physics, vol. 2012, no. 4, article 007, 2012.

[43] G. G. L. Nashed, "Charged axially symmetric solution and energy in teleparallel theory equivalent to general relativity," The European Physical Journal C, vol. 49, no. 3, pp. 851-857, 2007.

[44] K. Atazadeh and F. Darabi, " $f(T)$ cosmology via Noether symmetry," The European Physical Journal C, vol. 72, article 2016, 2012.

[45] H. Wei, X.-J. Guo, and L.-F. Wang, "Noether symmetry in $f(T)$ theory," Physics Letters B, vol. 707, no. 2, pp. 298-304, 2012.

[46] K. Karami and A. Abdolmaleki, "Holographic $f(T)$-gravity model with power-law entropy correction," Physical Review D, vol. 88, no. 8, Article ID 084034, 12 pages, 2013.

[47] P. A. Gonzalez, E. N. Saridakis, and Y. Vasquez, "Circularly symmetric solutions in three-dimensional Teleparallel, $f(T)$ and Maxwell- $f(T)$ gravity," Journal of High Energy Physics, vol. 2012, p. 53, 2012.

[48] S. Capozziello, V. F. Cardone, H. Farajollahi, and A. Ravanpak, "Cosmography in $f(T)$ gravity," Physical Review D, vol. 84, no. 4, Article ID 043527, 2011.

[49] R.-X. Miao, M. Li, and Y.-G. Miao, "Violation of the first law of black hole thermodynamics in $f(T)$ gravity," Journal of Cosmology and Astroparticle Physics, vol. 2011, article 033, 2011.

[50] X.-H. Meng and Y.-B. Wang, "Birkhoff's theorem in $f(T)$ gravity," The European Physical Journal C, vol. 71, article 1755, 2011.

[51] T. Shirafuji, G. G. Nashed, and Y. Kobayashi, "Equivalence principle in the new general relativity," Progress of Theoretical Physics, vol. 96, no. 5, pp. 933-947, 1996.

[52] H. Wei, X.-P. Ma, and H.-Y. Qi, " $f(T)$ theories and varying fine structure constant," Physics Letters B, vol. 703, no. 1, pp. 74-80, 2011.

[53] M. Li, R.-X. Miao, and Y.-G. Miao, "Degrees of freedom of $f(T)$ gravity," Journal of High Energy Physics, vol. 2011, no. 7, article 108, 2011.

[54] S. Chattopadhyay and U. Debnath, "Emergent universe in the chameleon, $f(R)$ and $f(T)$ gravity theories," International Journal of Modern Physics D, vol. 20, no. 6, pp. 1135-1152, 2011.

[55] P. B. Khatua, S. Chakraborty, and U. Debnath, "Dilaton dark energy model in $f(R), f(T)$ and hořava-lifshitz gravities," International Journal of Theoretical Physics, vol. 51, no. 2, pp. 405-417, 2012.

[56] R.-J. Yang, “Conformal transformation in $f(T)$ theories," Europhysics Letters, vol. 93, no. 6, Article ID 60001, 2011.

[57] D. Liu and M. J. Reboucas, "Energy conditions bounds on $f(T)$ gravity," Physical Review D, vol. 86, no. 8, Article ID 083515, 2012.

[58] K. Atazadeh and M. Mousavi, "Vacuum spherically symmetric solutions in $f(T)$ gravity," The European Physical Journal C, vol. 73, article 2272, 2013. 
[59] G. G. L. Nashed, "General regular charged space-times in teleparallel equivalent of general relativity," The European Physical Journal C, vol. 51, no. 2, pp. 377-383, 2007.

[60] R.-J. Yang, "New types of $f(T)$ gravity," The European Physical Journal C, vol. 71, article 1797, 2011.

[61] R.-J. Yang, "Conformal transformation in $f(T)$ theories," $E P L$ (Europhysics Letters), vol. 93, no. 6, Article ID 60001, 2011.

[62] S. Nesseris, S. Basilakos, E. N. Saridakis, and L. Perivolaropoulos, "Viable $f(T)$ models are practically indistinguishable from $\Lambda$ CDM," Physical Review D, vol. 88, no. 10, Article ID 103010, 2013.

[63] A. V. Kpadonou, M. J. S. Houndjo, and M. E. Rodrigues, "Tolman-Oppenheimer-volkoff equations and their implications or the structures of relativistic stars in $f(T)$ gravity," http://arxiv.org/abs/1509.08771.

[64] G. Abbas, A. Kanwal, and M. Zubair, "Anisotropic compact stars in $f(T)$ gravity," Astrophysics and Space Science, vol. 357, no. 2, article 109, 2015.

[65] R. Zheng and Q.-G. Huang, "Growth factor in $f(T)$ gravity," Journal of Cosmology and Astroparticle Physics, vol. 2011, no. 3, article 002, 2011.

[66] R. Ferraro and F. Fiorini, "Cosmological frames for theories with absolute parallelism," International Journal of Modern Physics: Conference Series, vol. 03, article 227, 2011.

[67] M. H. Daouda, M. E. Rodrigues, and M. J. S. Houndjo, "Static anisotropic solutions in $f(T)$ theory," European Physical Journal C, vol. 72, no. 2, pp. 1-12, 2012.

[68] M. Hamani Daouda, M. E. Rodrigues, and M. J. S. Houndjo, "Anisotropic fluid for a set of non-diagonal tetrads in $\mathrm{f}(\mathrm{T})$ gravity," Physics Letters B, vol. 715, no. 1-3, pp. 241-245, 2012.

[69] C. G. Böhmer, A. Mussa, and N. Tamanini, "Existence of relativistic stars in $f(T)$ gravity," Classical and Quantum Gravity, vol. 28, no. 24, Article ID 245020, 2011.

[70] G. G. L. Nashed, "A special exact spherically symmetric solution in $\mathrm{f}(\mathrm{T})$ gravity theories," General Relativity and Gravitation, vol. 45, no. 10, pp. 1887-1899, 2013.

[71] S. Capozzielloa, P. A. González, E. N. Saridakise, and Y. Vásquez, "Exact charged black-hole solutions in D-dimensional $f(T)$ gravity: torsion vs curvature analysis," Journal of High Energy Physics, vol. 2013, no. 2, article 039, 2013.

[72] A. Paliathanasis, S. Basilakos, E. N. Saridakis et al., "New Schwarzschild-like solutions in $f(T)$ gravity through Noether symmetries," Physical Review D-Particles, Fields, Gravitation and Cosmology, vol. 89, no. 10, Article ID 104042, 2014.

[73] G. G. L. Nashed, "Spherically symmetric charged-dS solution in $f(T)$ gravity theories," Physical Review D, vol. 88, no. 10, Article ID 104034, 2013.

[74] M. E. Rodrigues, M. J. S. Houndjo, D. Sáez-Gómez, and F. Rahaman, "Anisotropic universe models in $f(T)$ gravity," Physical Review D, vol. 86, no. 10, Article ID 104059, 2012.

[75] J.-T. Li, C.-C. Lee, and C.-Q. Geng, "Einstein static universe in exponential $f(T)$ gravity," The European Physical Journal C, vol. 73, article 2315, 2013.

[76] I. G. Salako, M. E. Rodrigues, A. V. Kpadonou, M. J. S. Houndjo, and J. Tossa, " $\Lambda C D M$ model in $f(T)$ gravity: reconstruction, thermodynamics and stability," Journal of Cosmology and Astroparticle Physics, vol. 2013, article 060, 2013.

[77] C. W. Misner, K. S. Thorne, and J. A. Wheeler, Gravitation, W. H. Freeman, San Francisco, Calif, USA, 1973.

[78] C. G. Böhmer, T. Harko, and F. S. N. Lobo, "Conformally symmetric traversable wormholes," Physical Review D. Particles,
Fields, Gravitation, and Cosmology, vol. 76, no. 8, Article ID 084014, 2007.

[79] C. G. Böhmer, T. Harko, and F. S. N. Lobo, "Wormhole geometries with conformal motions," Classical and Quantum Gravity, vol. 25, no. 7, Article ID 075016, 2008.

[80] G. G. L. Nashed, "Vacuum non singular black hole solutions in tetrad theory of gravitation," General Relativity and Gravitation, vol. 34, no. 7, pp. 1047-1058, 2002.

[81] S. W. Hawking and G. E. R. Ellis, The Large Scale Structure of Spacetime, Cambridge University Press, Cambridge, UK, 1973.

[82] S. Carroll, Spacetime and Geometry: An Introduction to General Relativity, Addison-Wesley, Reading, Mass, USA, 2004.

[83] M. Zubair and S. Waheed, "Energy conditions in $f(T)$ gravity with non-minimal torsion-matter coupling," Astrophysics and Space Science, vol. 355, pp. 361-369, 2015.

[84] L. Herrera, "Cracking of self-gravitating compact objects," Physics Letters A, vol. 165, no. 3, pp. 206-210, 1992.

[85] P. Bhar, F. Rahaman, S. Ray, and V. Chatterjee, "Possibility of higher-dimensional anisotropic compact star," The European Physical Journal C, vol. 75, no. 5, 2015.

[86] A. Das, F. Rahaman, B. K. Guha, and S. Ray, "Relativistic compact stars in $f(T)$ gravity admitting conformal motion," Astrophysics and Space Science, vol. 358, article 36, 2015.

[87] R. Ruderman, "Pulsars: structure and dynamics," Annual Review of Astronomy and Astrophysics, vol. 10, pp. 427-476, 1972.

[88] N. K. Glendenning, Compact Stars: Nuclear Physics, Particle Physics and General Relativity, Springer, New York, NY, USA, 1997.

[89] M. Herjog and F. K. Röpke, "Three-dimensional hydrodynamic simulations of the combustion of a neutron star into a quark star," Physical Review D, vol. 84, no. 8, Article ID 083002, 2011. 

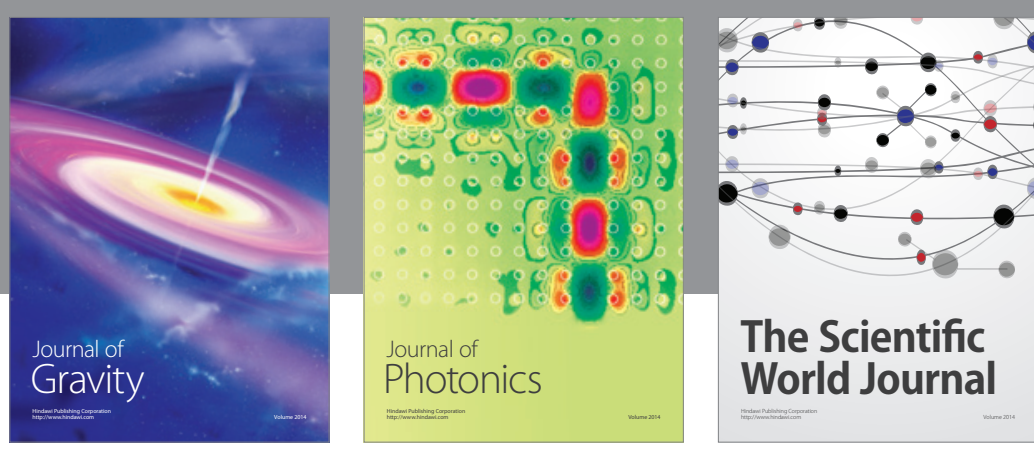

The Scientific World Journal
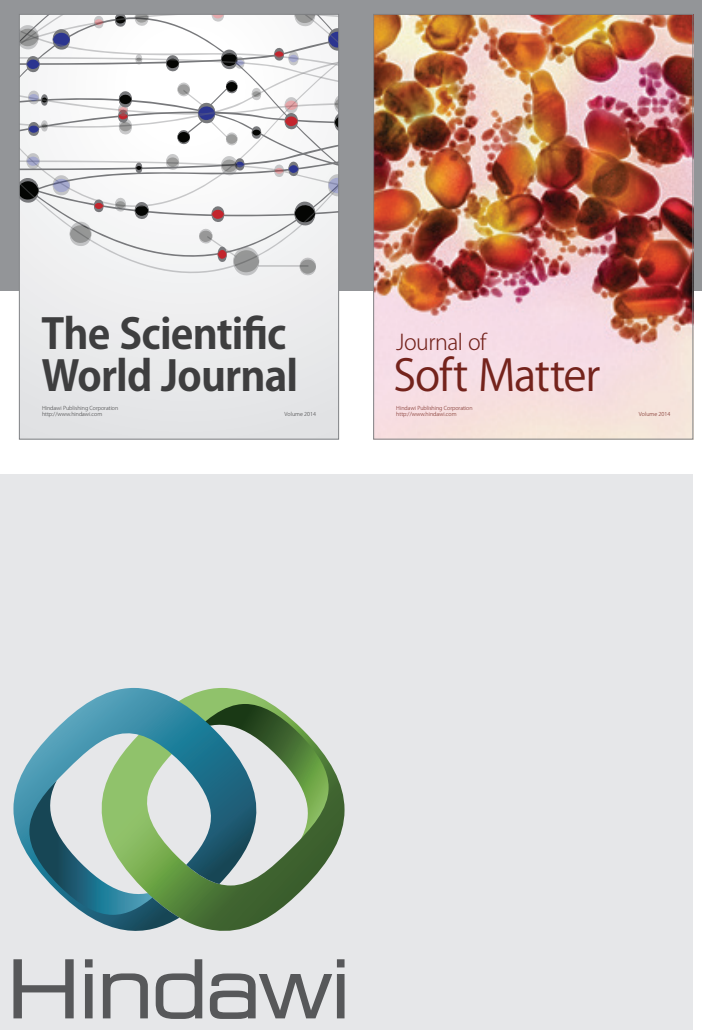

Submit your manuscripts at

http://www.hindawi.com

nternational Journal of

Statistical Mechanics
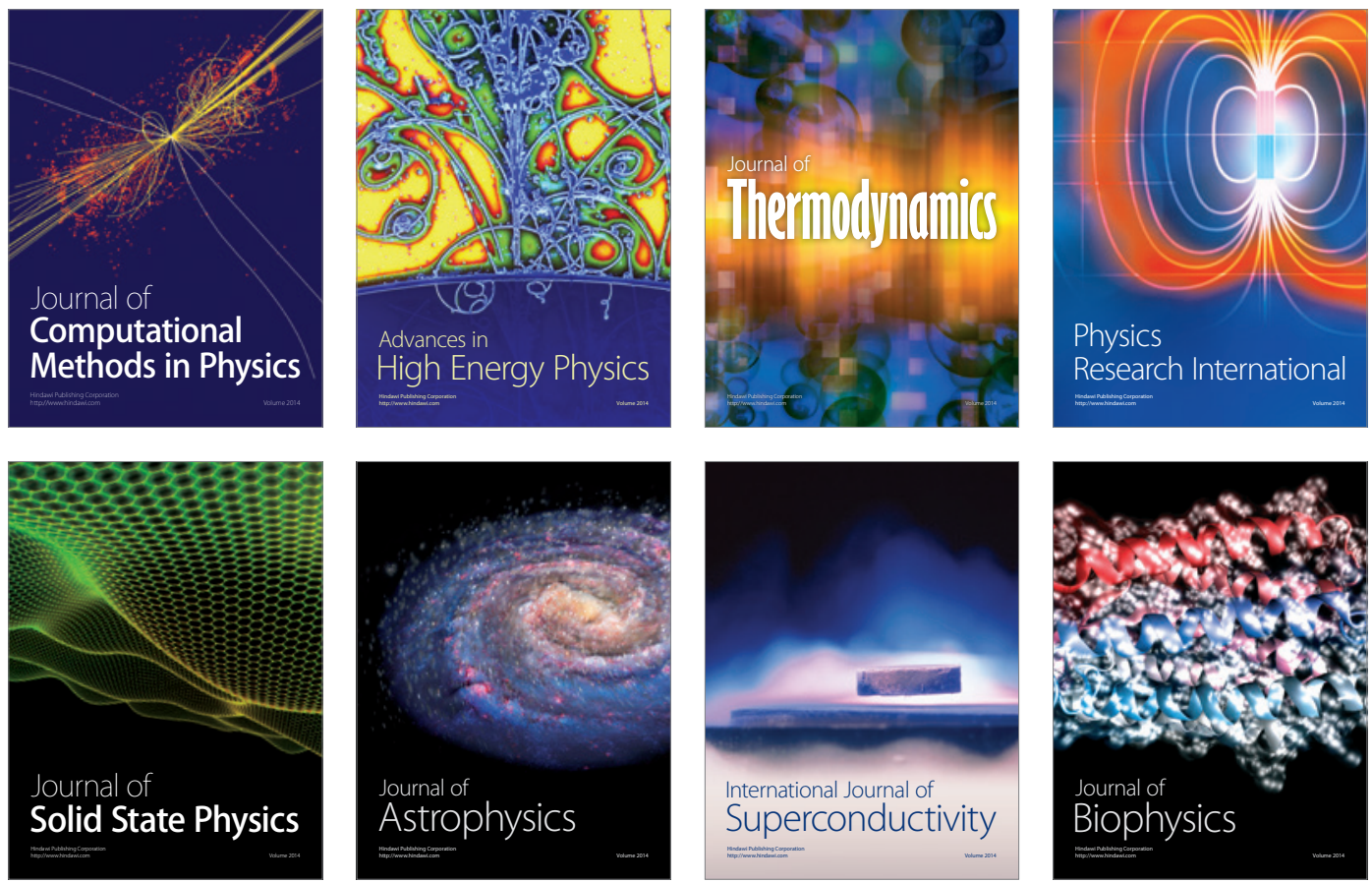
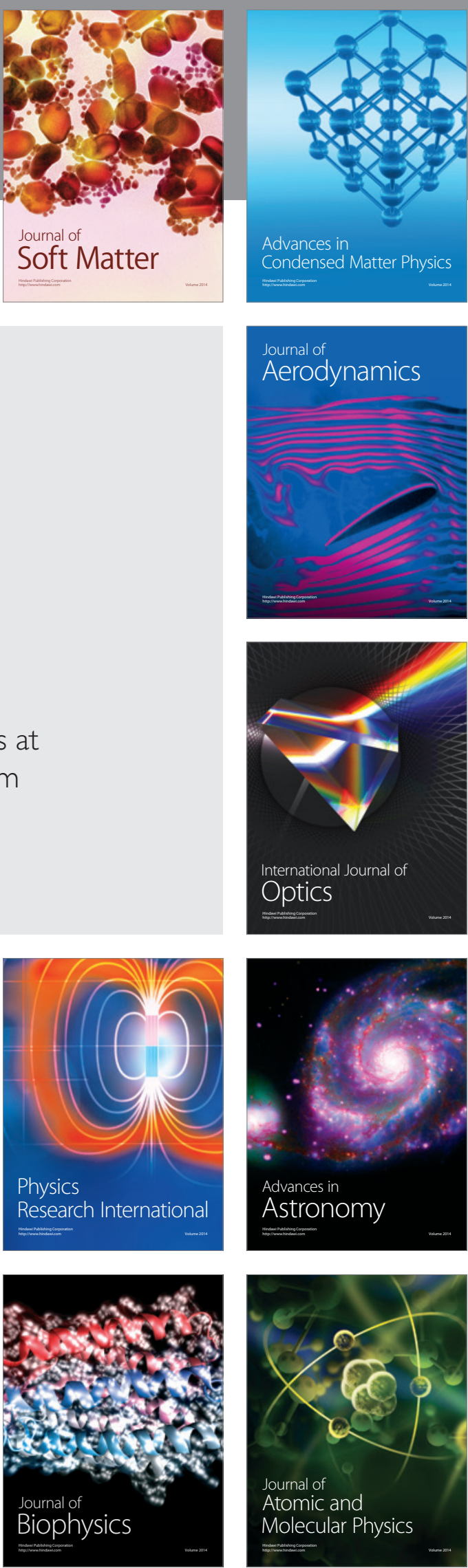